\title{
THE USE OF HETEROLOGOUS ANTILYMPHOID AGENTS
}

\section{IN CANINE RENAL AND LIVER HOMOTRANSPLANTATION AND IN HUMAN RENAL HOMOTRANSPLANTATION}

\author{
THOMAS E. STARZL, M.D., F.A.C.S., and THOMAS L. MARCHIORO, M.D., F.A.C.S., \\ Denver, Colorado, K. A. PORTER, M.D., London, England, YOJI IWASAKI, M.D., \\ Chiba, Japan, and G. JAMES CERILLI, M.D., Denver, Colorado
}

The Potential VALUe of heterologous antilymphoid serum as an immunosuppressive agent has been known for a number of years. However, the first reference to testing of antiserums for homograft protection was by Woodruff, who later published with Anderson $(20,21)$ detailed observations of the effect of these agents used alone or in combination with thoracic duct drainage. Subsequent valuable studies have been contributed by Waksman, Gray (2), Monaco $(9,10)$ and their respective associates and by Jeejeebhoy, Nagaya and Sieker, and Levey and Medawar $(6,7)$. The experimental model for the foregoing experiments consisted of skin grafting in mice, rats, or guinea pigs which were treated with antiserums raised in rabbits. Except for our own brief report (14) and those of Abaza, and Mitchell and their colleagues there are no published accounts on the use of antilymphoid serum for the protection of whole organ homografts.

The present study is a further evaluation of the importance of this kind of immunosuppressive therapy for the protection of whole organ homografts in dogs, as well as an account of its subsequent application in humans. Antiserums against the lymphoid tissue of both species were raised in horses,

From the Department of Surgery, University of Colorado School of Medicine and the Denver Veterans Administration Hospital, Denver, and the Department of Pathology, St. Mary's Hospital and Medical School, London.

Aided by U. S. Public Health Service grants AM 06283, Aided by U. S. Public Health Service grants AM 06283,
AM 06344, HE 07735, AM 07772, AI 04152, FR 00051, and FR 00069 and by a grant from the Medical Research Council of Great Britain. characterized for toxicity and antibody content, and rendered to globulin as fully described elsewhere (4). The test organs in the canine experiments were the kidney and liver. The clinical patients had renal homotransplantation.

\section{METHODS}

Canine experiments. One hundred and thirty-four mongrel dogs weighing 10 to 25 kilograms were immunized against hepatitis and distemper and used as homograft recipients. Operations were performed under sodium pentobarbital anesthesia combined with the tranquilizer phencyclidine hydrochloride. Renal transplants were accomplished by transferring the donor kidney to the contralateral recipient iliac fossa, with anastomoses of the renal artery to the proximal end of the cut common iliac artery, the renal vein to the side of the common iliac vein, and the ureter to the bladder. Bilateral recipient nephrectomy was always carried out. Orthotopic hepatic transplants were performed as previously described (15). Before and after both kinds of homotransplantation, blood urea nitrogen, serum bilirubin, alkaline phosphatase, serum glutamic oxalacetic transaminase, serum glutamic pyruvic transaminase, and complete hematologic studies were obtained at frequent intervals. The development of canine antibodies against horse serum was monitored by determination of precipitin titers (4).

Animals which died after transplantation were autopsied and the tissues examined by

Reprint from SURGERY, Gynecology 60 Obstetrics, February, 1967, Vol. 124, 301-318

Copyright, 1967, by The Franklin H. Martin Memorial Foundation 


\section{Surgery, Gynecology \& Obstetrics · February 1967}

light microscopy. In a number of experiments, biopsies were taken at varying intervals after transplantation and examined both with light and electron microscopy.

Antilymphoid plasma.-The 3 kinds of antilymphoid agents were obtained from immunized horses as described previously (4). The first was unabsorbed antilymphoid plasma with a leukoagglutinin titer of 1:16 to $1: 256$. The dose of 1 to 4 milliliters per kilogram was given intraperitoneally every 1 to 3 days for 5 to 30 days before renal homotransplantation and by a similar schedule during the first 20 or 30 postoperative days. After this time, the intervals between injections were increased to 4 to 30 days or therapy was stopped. The plasma was toxic. Of 36 dogs entered into the experiment, 11 died before operation. Twelve of the remaining 25 definitive test animals had thymectomy 7 to 20 days before institution of plasma therapy.

Antilymphoid serum.-The second product tested was antilymphoid serum with a titer of $1: 32$ to $1: 128$. This was obtained from 4 of the same horses, but it was prepared from coagulated blood and partially absorbed against 10 per cent dog red cell pack. Six dogs received renal homotransplantation with a comparable intraperitoneal dose schedule as that described for plasma. In addition, 6 other animals received kidneys with in traperitoneal antiserum therapy starting on the day of transplantation.

The same antilymphoid serum was given intraperitoneally to 9 recipients of orthotopic liver homografts, beginning 1 to 26 days before operation. One of the pretreated dogs received no further therapy after transplantation, but the others had additional postoperative injections.

Antilymphoid globulin.-The third kind of antilymphoid agent tested was crude globulin which was precipitated from immune horse serum with ammonium sulfate after absorption of the serum with dog red cells, and in some instances with dog serum, kidney, and liver. Many of the batches used were concentrated by lyophilization.
The leukoagglutinating titer of the reconstituted injectate was $1: 512$ to $1: 1,024$. The subcutaneous dose schedule was 0.2 to 0.5 milliliter per kilogram per day. The effectiveness of immunosuppression was evaluated both with renal and orthotopic liver homotransplantation.

For 25 kidney transplantations pretreatment of 7 to 13 days was provided for 12 , and therapy was begun on the day of operation in the other 13. In 4 of 9 dogs receiving liver grafts therapy was started on the day of transplantation, and the other 5 had 5 to 26 days of pretreatment. In the recipients of both kidney and liver homografts, therapy was stopped from 35 to 60 days after transplantation.

Another 4 dogs received renal homotransplantation after pretreatment for 60 days. No postoperative therapy was given to these animals.

Antilymphoid globulin and azathioprine. - Crude globulin was given to 14 dogs, beginning on the day of operation. Postoperatively, 1 milligram per kilogram per day azathioprine was added.

Control studies. - The survival of nontreated control animals previously reported from our laboratory was $11.3 \pm 4.6$ standard deviation (SD) days after renal homotransplantation (14) and $7.1 \pm 2.2$ (SD) days after orthotopic liver transplantation (15). New control animals were prepared for the present study with 7 renal and 10 orthotopic liver transplantations. The results were not different from those in the older series for which reason both old and new groups were combined for statistical comparison with the results using antilymphoid therapy.

Four other control animals were treated for 30 days with crude globulin prepared from the serum of nonimmunized horses. The dose of protein was the same, approximately 30 milligrams per kilogram per day, as with the globulin from immunized horses. Renal homotransplantation was then performed, and the subcutaneous globulin administration continued daily until death. 
Renal homotransplantation was performed in 7 dogs treated only with 1 milligram per kilogram per day azathioprine.

Data comparison.-The various operations were performed over a period of almost $11 / 2$ years with variable periods of available follow-up. In order to avoid bias in the event of unusually long survival and to allow comparison of series studied at different times, credit for survival of any animal was limited to 70 days.

The variously tested antilymphoid substances differed in their titer, degree of purification, and extent of absorption with tissues or red cells from the lymphoid donor species. Therefore, an effort was made to relate the results by means of a unit system which was based upon leukoagglutinating titers of these different agents. If 1 milliliter of either horse plasma, horse serum, or crude horse globulin had a titer of $1: 128$, it was said to contain 128 units. With a titer of $1: 1,024,1$ milliliter was considered to contain 1,024 units. It is recognized that such a unit nomenclature is arbitrary and that the therapeutic efficacy may not be directly related to the titer as the system implies.

Clinical studies. Twelve patients have been given intramuscular antihuman immune globulin which was prepared from the serum of horses immunized with the lymph nodes, thymuses, and spleens of fresh cadavers (4). After absorption of the horse serum with human red cells and serum, precipitation with ammonium sulfate, and lyophilization, the reconstituted product had a leukoagglutinin titer of from $1: 8,192$ to $1: 16,384$. The single doses of 4 milliliters provided 450 to 2,100 units per kilogram. The protein content was 7 to 9 grams per cent. The development of human antibodies against horse protein was monitored serially with precipitin tests. The titers of hemagglutinins against sheep red blood cells were also followed, and intradermal skin tests with 0.1 milliliters injectate were performed every 2 weeks. The response to antilymphoid globulin of nontransplanted uremic individuals is described elsewhere (4).
Eight patients with terminal renal failure were treated with immune globulin. In 7 , the horse globulin was given for 5 or 6 days in advance of renal homotransplantation as well as postoperatively. In the eighth patient, pretreatment was for 35 days. After operation, injections were continued daily for 1 to 2 weeks, then every other day for 2 to 3 weeks, and finally reduced to twice a week until the fourth month after which 1 injection per week was given. Two of these patients had transthoracic thymectomy prior to transplantation. All 8 kidneys were obtained from blood relatives. There were 2 mothers and 6 siblings. The donor and recipient ages ranged from 21 to 45 and from 16 to 36 years respectively.

In contrast to the animal experiments, horse globulin was never used as the sole immunosuppressive agent. Azathioprine was started on the day of operation and continued thereafter in daily doses of 5.2 to 0.5 milligram per kilogram per day. During the first month, prednisone was added if needed for control of rejection. After this time, when the antilymphoid globulin therapy was being reduced, prednisone was started in doses of 0.3 to 0.67 milligram per kilogram per day for patients who had not had rejection. Actinomycin $\mathrm{C}$ in intravenous doses of 400 micrograms and local homograft irradiation were used irregularly if and when rejection occurred.

To evaluate the effect of the globulin therapy, comparisons were made with comparable patients treated in the past at the University of Colorado in whom survival of at least 63 days had been attained. The control patients of intrafamilial homotransplantations were divided into 3 groups of 25,13 , and 22 each. The first series of recipients, treated in 1962 and 1963, had received azathioprine only until the onset of rejection at which time prednisone was added. The second group received high dose steroid therapy from the day of operation onward in addition to azathioprine. The third group differed in that smaller quantities of prednisone, usually 0.5 milli- 


\section{Surgery, Gynecology \& Obstetrics · February 1967}

gram per kilogram per day, were started on the day of operation. This steroid dose was increased only when required to treat rejection. In all 3 groups, attempts were later made to reduce the prednisone dosage as rapidly as possible. Since inclusion in the control groups was contingent upon survival for 63 days, unfavorable patients were automatically eliminated. Thus 7,1 , and 3 patients who were unsuccessfully treated with the respective protocols for less than this period were excluded. An additional patient in group 2 died on the last day but is included.

Lymphocyte antigens were analyzed by Terasaki and his associates in the donors and recipients from the 3 control groups as well as the antilymphoid series. This information became available retrospectively in 21 of the 25 patients of group 1 and 10 of the 13 patients in group 2. It was obtained prospectively for all patients of group 3 and of the antilymphoid series. In the last series, there were good, average, and poor matches in 3, 3 , and 2 patients respectively. Statistical comparison performed by Terasaki revealed no significant differences in the quality of antigen compatibility among the donor-recipient combinations in 4 groups.

For each of the 3 control groups and for the antilymphoid series, the events in the first 63 postoperative days were compared by determining the average daily blood urea nitrogen, creatinine, creatinine clearance, white blood count, lymphocyte differential, lymphocyte count, azathioprine dose, and prednisone dose. From these data it could be determined if the function in the antilymphoid patients was as good as in the previous series, if this function had been achieved with greater or smaller doses of standard immunosuppressive agents, and if there were consistent differences in the peripheral white cell differential and total counts in the various groups.

Late homograft rejection.-Three patients had received maternal, cadaveric, and maternal kidneys respectively, 11, 7, and 5 months previously. In each, there was de- terioration of renal function when the prednisone dose was reduced below 1.3, 1.7, and 1 milligram per kilogram per day, in the first, second, and third patients. In each instance, 4 milliliters of antilymphoid globulin with a titer of $1: 4,096$ to $1: 16,384$ were instituted every other day or twice a week, and the prednisone dose was then attenuated.

Secondary cadaveric homotransplantation.-A 23 year old girl with removal of a failed homograft 31 months after transplantation from a nonrelated living donor received 2 cadaveric kidneys 5 and 8 weeks later while being treated with antilymphoid globulin, azathioprine, and prednisone. The first cadaveric homograft which began to excrete urine after a week was removed as an emergency 2 days later because of acute renal vein thrombosis. She had a cardiac arrest during bronchoscopy and died 8 days after placement of the second cadaveric kidney which did not function. Since evaluation of therapy was impossible under these circumstances, this patient will not be discussed further.

\section{RESULTS}

Canine experiments. Antilymphoid plasma. - The 25 dogs treated before and after renal homotransplantation with doses of 64 to 512 units immune plasma had a survival of 28.2 \pm 22 (SD) days, a significant $(\mathrm{p}<0.01)$ improvement over that of controls. The mean figures are somewhat lower than those previously reported (14) because survival credit for any individual animal was limited to 70 days. The 12 dogs with thymectomy lived for $26.4 \pm 22$ (SD) days compared to $29.8 \pm$ 24 (SD) days for the other 13, a difference which was not significant. Of the 25 animals, $18,11,9$, and 6 survived for at least 15, 20, 30 , and 50 days respectively. The 2 longest survivors lived for 95 and 144 days.

During the early postoperative period when injections were given every 1 to 3 days, the animals lost weight. They usually became profoundly anemic, a complication apparently attributable at least in part to the high hemagglutinin titers in the immune plasma 


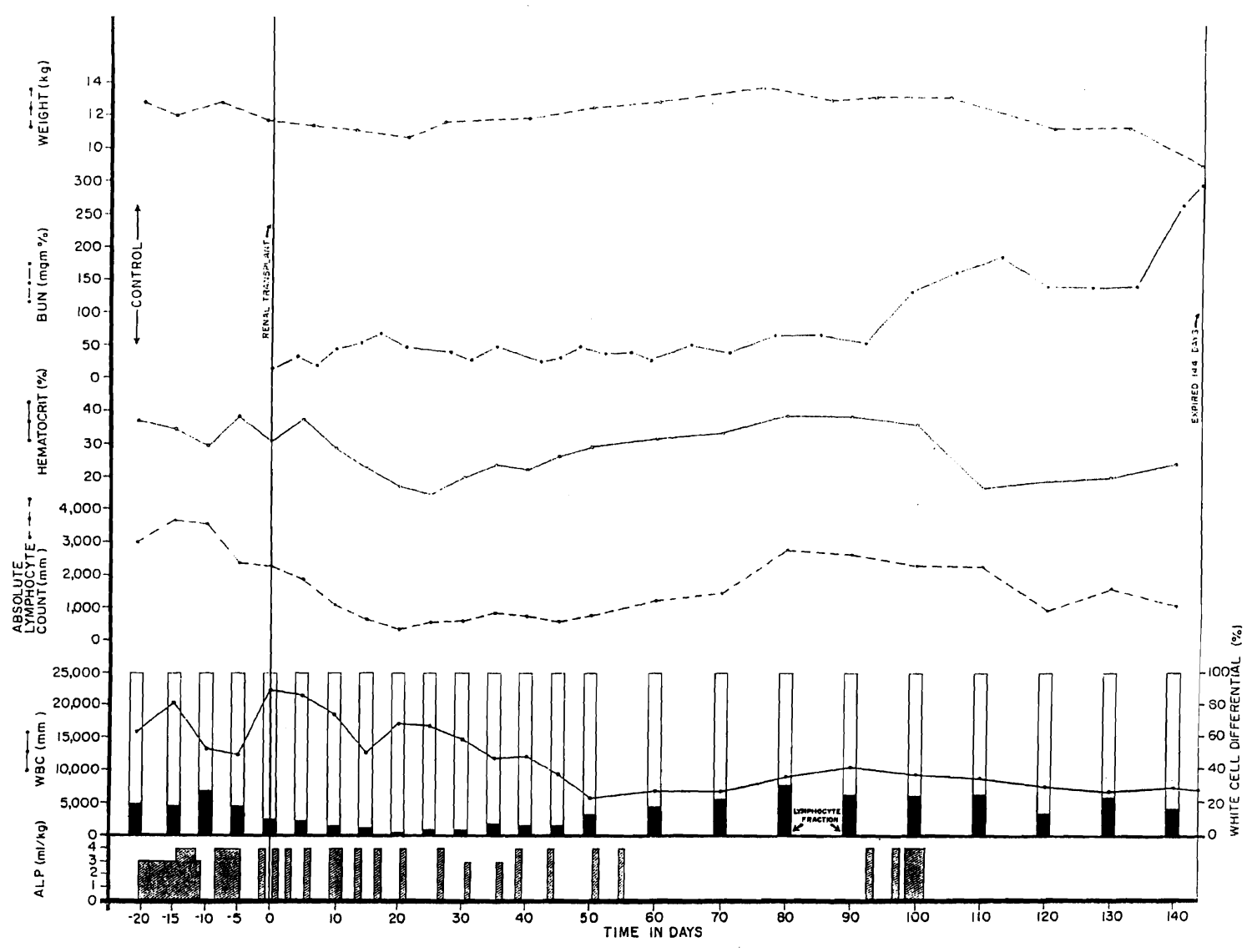

Fig. 1. Course of a dog after renal homotransplantation, employing antilymphoid plasma ( $A L P$ ) for immunosuppression. Note the weight loss and anemia caused by both an early and late course of therapy. Rejection was slow to occur after cessation of treatment. Note the minimal change in the lymphocyte count when the plasma was given during the preoperative period.

(4). Later when therapy was either reduced or stopped, renal failure developed, usually rather slowly, in the longer surviving animals (Fig. 1).

Antilymphoid serum.-Weight loss and anemia were also observed with intraperitoneal antilymphoid serum administrations, but there were no preoperative deaths. The 6 dogs with renal homotransplantation which received both preoperative and postoperative serum in doses of 64 to 512 units survived $27.2 \pm 13$ (SD) days, a statistically significant $(\mathrm{p}<0.01)$ immunosuppressive effect. Five, 4, and 2 animals lived at least 15,20 , and 30 days respectively. The longest survival was 49 days.

In contrast, although 1 of the 6 animals treated beginning at the time of renal homo- transplantation lived 38 days, the others all died in less than 15 days. Mean survival was $12.8 \pm 12.5$ (SD) days, a value which was not significantly better than nontreated controls.

After orthotopic liver transplantation 5, 4, 3 , and 2 of the 9 animals lived for more than $15,20,30$, and 50 days respectively. The mean survival, with a limitation of maximum survival credit to 70 days for individual dogs, was $26.8 \pm 26$ (SD) days, indicating a statistically significant $(\mathrm{p}<0.05)$ prolongation of life. Moreover, 2 of the animals are still alive and in good health after 5 and 6 months.

Two important observations were made in these 2 long-surviving dogs. One animal received only 6 injections of antilymphocyte 
6 Surgery, Gynecology \& Obstetrics · February 1967

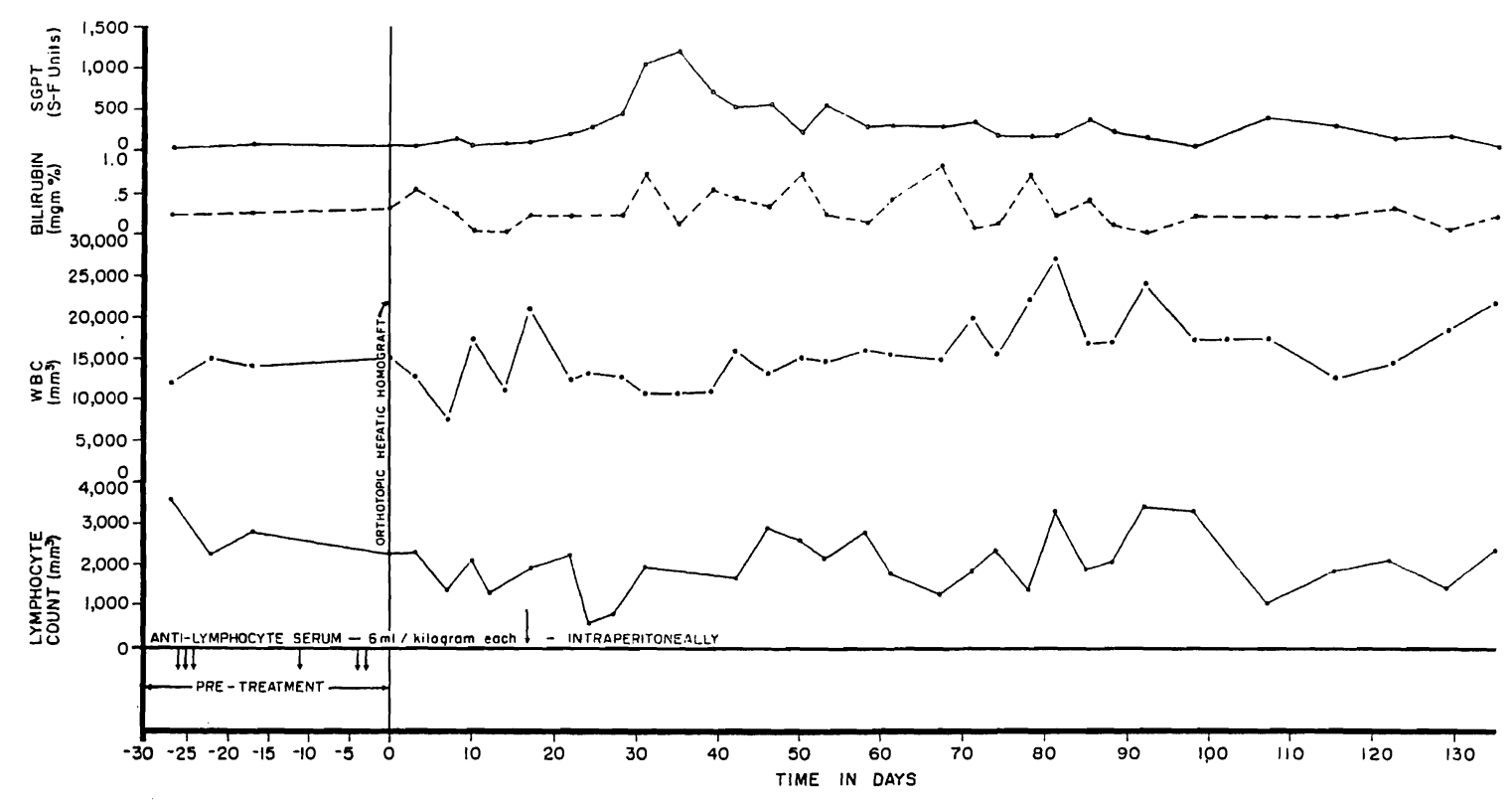

Fig. 2. A dog which received an orthotopic liver homograft after 6 intraperitoneal injections of antilymphoid serum $(A L S)$. No postoperative therapy was ever given. Note that the lymphocyte count was little changed. The dog is still alive.

serum over a 26 day preoperative period. Afterliver transplantation, no further therapy was given. A definite lymphopenic effect was not produced (Fig. 2). The second animal, which had pretreatment for 1 month, received postoperative serum treatment for only 20 days. After stopping therapy, there was a striking lymphocytosis (Fig. 3), despite which overt rejection has not occurred.

Antilymphoid globulin. - The dogs received 100 to 500 units of globulin subcutaneously. Gradual weight loss was not uncommon. A few batches of globulin caused anemia which developed slowly over a period of several weeks, but this was an unusual complication.

The 12 dogs treated both before and for 2 months after renal homotransplantation had a postoperative survival of $22.9 \pm 23$ (SD) days, a statistically significant $(\mathrm{p}<0.02)$ prolongation of survival. Five, 4,3 , and 3 animals lived for more than 15,20,30, and 50 days respectively. In contrast, the 13 dogs treated only after operation had a reduced survival of $14.3 \pm 7$ (SD) days, a prolongation which was nevertheless statistically significant $(\mathrm{p}<0.05)$. This group had $4,2,1$, and 0 living animals after 15,20,30, and 50 days. The slow but progressive late rejection in the animals treated with globulin (Fig. 4) was similar to that described earlier in recipients treated with immune plasma or serum.

The 4 dogs which received kidneys after 60 days of pretreatment but which were not treated after operation all died after 9 to 15 days. Survival was $12 \pm 3$ (SD) days. There was no statistically significant difference from nontreated controls.

The immunosuppressive effect after orthotopic liver transplantation was more consistent $(p<0.01)$. With limitation of credit to a maximum of 70 days for any dog, the mean survival was $36 \pm 30(\mathrm{SD})$ days. Five, 5,4 , and 4 dogs lived for more than 15, 20, 30 , and 50 days respectively. The longest survival, in an animal which is still alive, has been 120 days. The 5 dogs which received therapy both before and after operation did not fare better than the 4 treated only afterward.

As described earlier with immune plasma or serum, the production or maintenance of lymphopenia was not a prerequisite for po- 


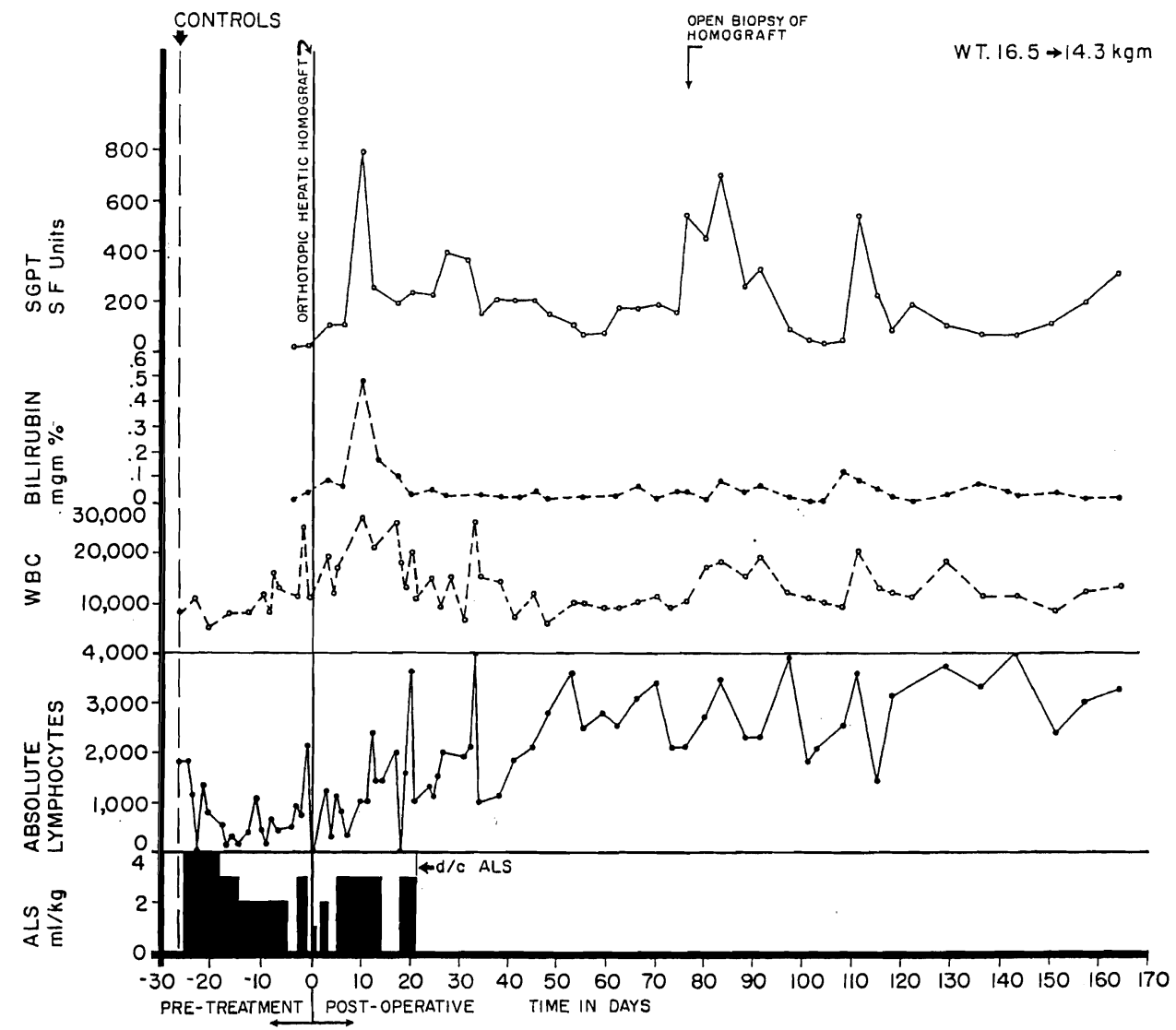

Fra. 3. A chronically surviving dog which was treated before and for 20 days after orthotopic liver transplantation with intraperitoneal antilymphoid serum $(A L S)$. Note the pronounced lymphocytosis late in the postoperative period. The animal is in excellent health after almost 6 months.

tentiation of survival after either kidney or liver homotransplantation (Fig. 4). There was not an apparent correlation between the presence or absence of postoperative lymphopenia and the success or failure of therapy.

Antilymphoid globulin and azathioprine. - The 14 dogs which received combined therapy lived for $22.4 \pm 19$ (SD) days, showing a statistically significant immunosuppressive effect $(p<0.01$ ). Eight, 5, 2, and 2 animals survived for more than $15,20,30$, and 50 days respectively. One of the 2 most chronic dogs died after 60 days and the other is still alive after 100 days. In subsequent statistical comparisons, it should be recalled that globulin therapy was started just before or at the time of operation.

Control studies. - The 7 untreated dogs with renal homotransplantation lived for 9.4 \pm 3 (SD) days, with a range of 5 to 14 days. These results, combined with those from 16 comparable experiments performed at an earlier time, gave a mean survival of $10.7 \pm$ 3 (SD) days. After orthotopic liver transplantation in 10 dogs, survival was $6.8 \pm 3$ (SD) days with a range of 2 to 11 days. The survival of these 10 fresh controls plus 22 more from earlier experiments was $7.0 \pm 3$ (SD) days.

The 4 animals treated preoperatively and postoperatively with globulin obtained from normal horse serum lived for $14.8 \pm 4$ (SD) days, with a range of 9 to 18 days. This survival was not significantly better $(p<0.4)$ than that in control animals.

The 7 dogs given 1 milligram per kilogram per day azathioprine survived $20.1 \pm 18$ (SD) days. Four, 2, 1, and 1 animals lived 


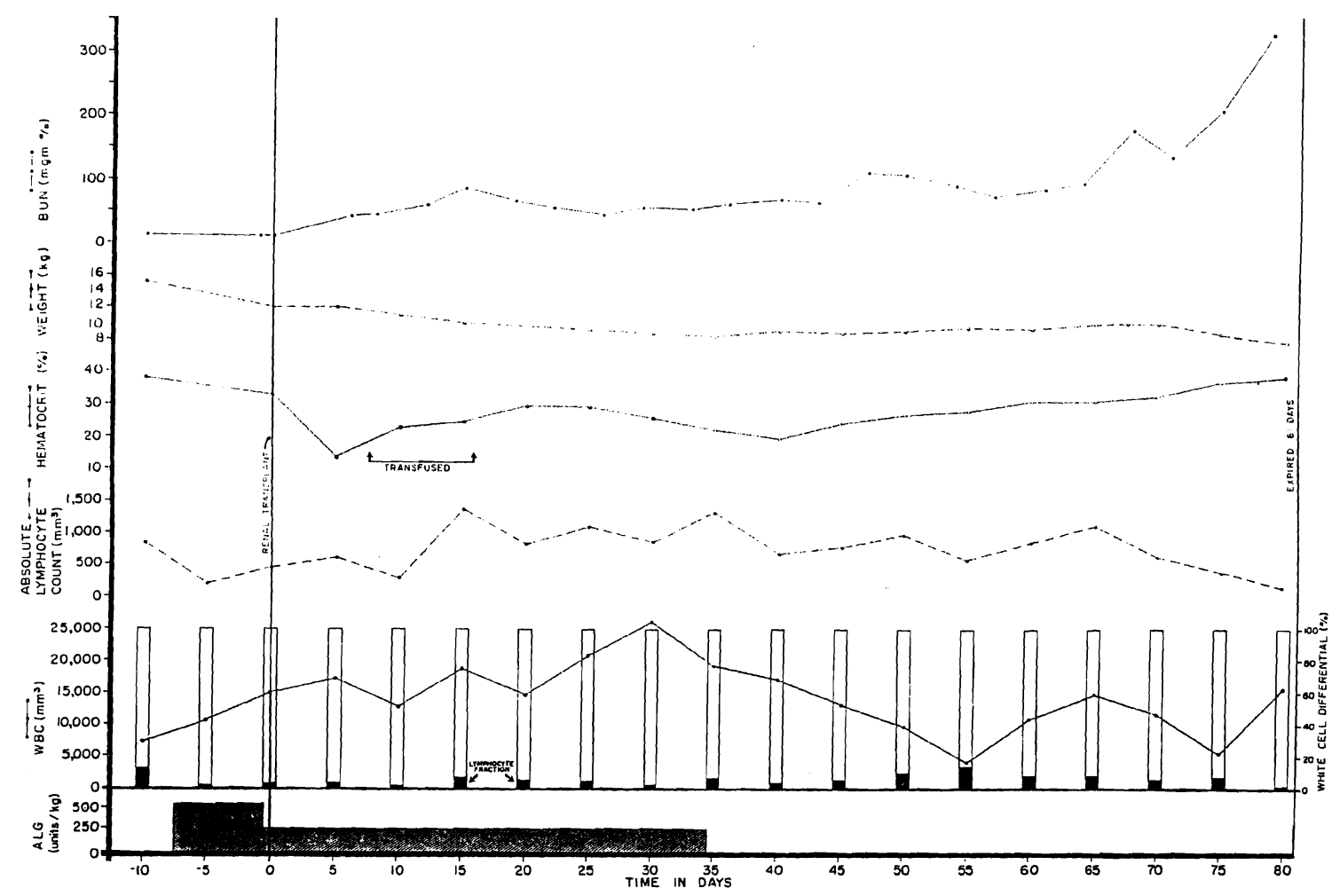

FIg. 4. Potentiation of canine renal homograft survival with immunosuppression by antidoglymphoid globulin $(A L G)$. Although the lymphocyte differential fraction was reduced, the total white count was increased and the absolute lymphocyte count was higher postoperatively than during the control period. The early postoperative anemia was apparently due to operative blood loss. Note the slow rejection after cessation of therapy.

for at least 15, 20,30, and 50 days respectively. Thus the doses, although small, had a statistically significant immunosuppressive effect $(p<0.05)$.

Data comparison. - The foregoing data were used to ask several questions of potential clinical importance. To do this, smaller groups were combined into larger series and the significance of differences in results were subjected to the Student t test.

Is pretreatment valuable? The 18 animals which received either antilymphoid serum or globulin both before and after transplantation had" a significantly ( $p<0.05)$ better survival than those 19 dogs treated only after operation.

Is combination therapy helpful or harmful? The survival of the 14 dogs treated postoperatively with 1 milligram per kilogram per day azathioprine plus immune globulin was compared with that of the 7 dogs which received the same course of azathioprine alone and with that of the 13 dogs which received only postoperative immune globulin. Although the results with the two combined agents were better than those obtained when antilymphoid globulin was used alone in a comparable dose schedule, the difference was not statistically significant $(p<0.2)$. Similarly, the increased survival with the two combined agents in comparison with that using azathioprine alone was not statistically significant.

Is success easier to attain with liver than with kidney transplantation? Survival in the 18 liver experiments involving antilymphoid serum or globulin was significantly better $(p<0.05)$ than in 37 comparable experiments with renal homotransplantation. The comparison has validity since approximately 


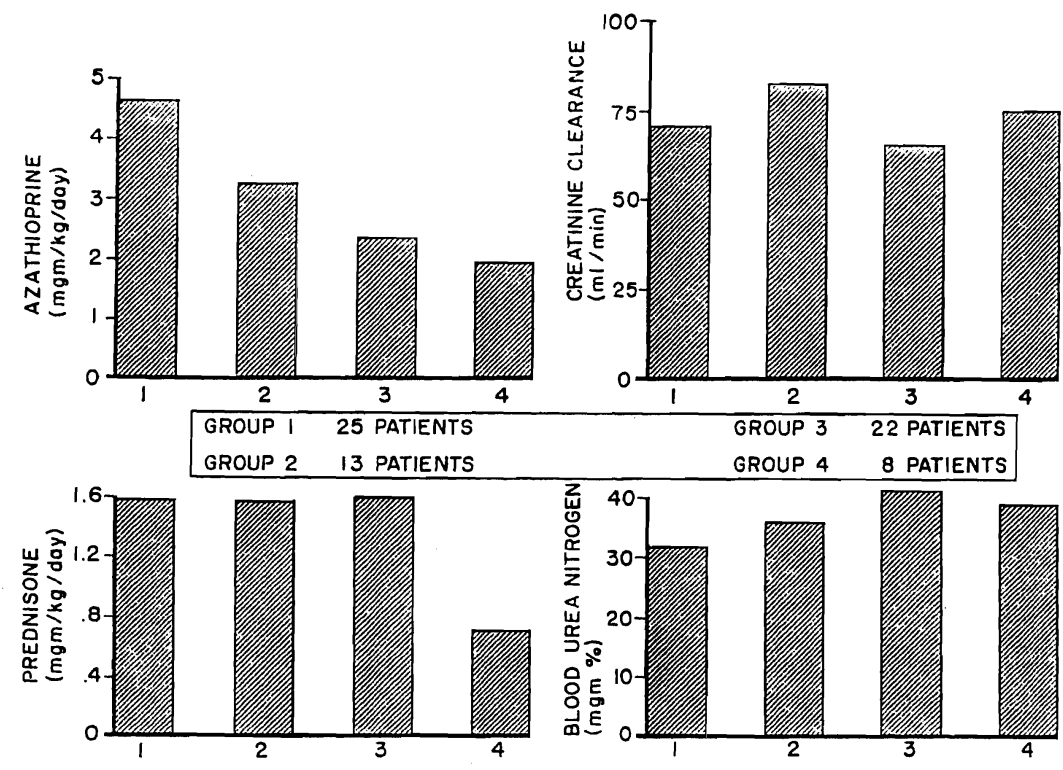

FIG. 5. Variations in immunosuppression and renal function during the first 63 postoperative days in 4 successive groups of patients who received kidneys from blood relatives. Since the blood urea nitrogen and creatinine clearance were not determined each day, these were compiled on a weekly basis. Those in series 4 received adjuvant therapy with antilymphoid globulin. Note the drastic reduction in average prednisone dose which was achieved in these patients without significant loss of renal function. The progressive diminution of azathioprine dosage in the succeeding series is evident.

half of the recipients in each kind of transplantation received therapy both before and after operation, and the other half were only treated following operation.

Pathologic studies. - Ninety-nine of the renal homografts were examined histologically. In dogs treated with antilymphoid substances the features of rejection were not different from those described by Porter and his associates after immunosuppression with other antirejection agents. These included infiltration with mononuclear cells of which 20 to 80 per cent had pyroninophilic cytoplasm. Platelet thrombi and intimal fibrous thickening of interlobular and arcuate arteries were common. Fibrinoid necrosis of arteriolar and arterial walls was present in half the specimens and glomerular capillary basement membrane thickening was found in 27 per cent. Vascular and glomerular changes were rare in either the controls or treated dogs with short survival. The only clear difference in the various test groups was a reduction in the degree of cellular infiltration, the number of cells possessing pyroninophilic cytoplasm, and the incidence of mitoses in those homografts from dogs treated with both low doses of azathioprine and antilymphoid globulin. Two of these 14 kidneys had no evidence of rejection, the only ones completely spared in the entire study.

Similarly, in the 17 hepatic homografts studied either from autopsy or biopsy specimens, the findings were comparable to those previously reported in dogs protected with azathioprine (15). Animals dying before 3 weeks had centrizonal and usually midzonal necrosis, infiltration of mononuclear cells around the portal tracts and central veins, and centrilobular cholestasis. The 6 homografts examined after 3 weeks had variable centrilobular hepatocyte atrophy or reticulin condensation, or even later fibrosis and proliferation of bile ductules in the portal tracts. Five of these 6 livers contained mononuclear cells but in 2 of these the numbers were low. 
10 Surgery, Gynecology \& Obstetrics · February 1967

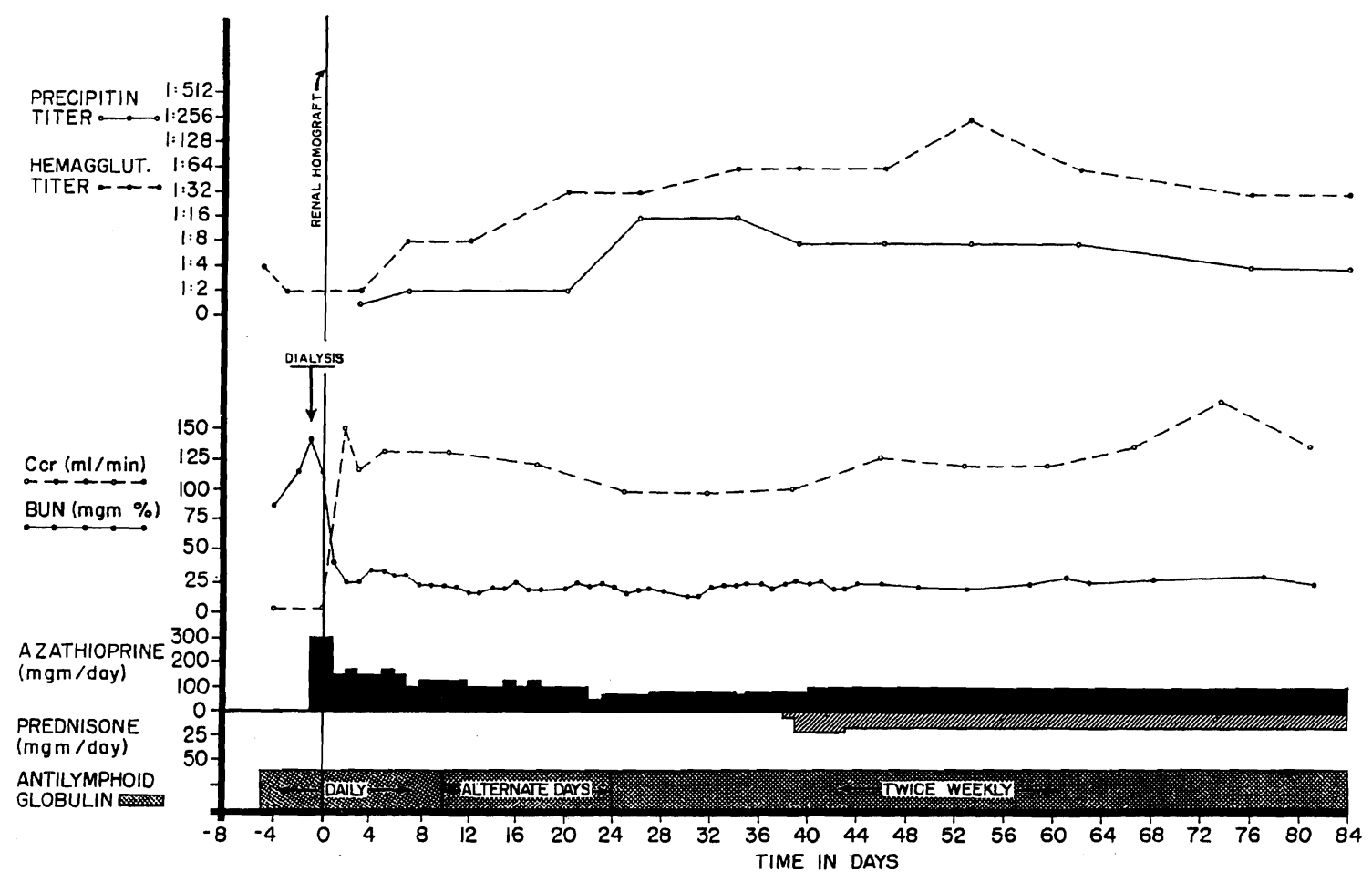

Fic. 6. Course of a patient treated before and after renal homotransplantation with antilymphoid globulin. No rejection occurred. Note the rises in precipitin and hemagglutinin titers, findings which prompted institution of prednisone therapy. These titers subsequently fell. This patient had a good antigen match with his sibling donor.

In all, pyroninophilic cells were sparse. In several of these livers, active rejection appeared to have ceased in contrast to the findings in the homografts of the longest surviving animals after renal homotransplantation when the histologic appearance suggested that none of these kidneys was being tolerated chronically.

The effect of the antilymphoid products on the lymphoid tissue, kidneys, and other recipient organs has been described (4). This included myocardial necrosis and necrotizing coronary arteritis which were also present but in lower incidence in the control animals. Dogs which had renal homotransplantation under antilymphoid therapy alone had hepatic cholestasis or centrizonal necrosis in an incidence of 10 and 4 per cent respectively. When azathioprine was also given, these figures rose to 77 and 61 per cent. This latter finding was probably largely due to the azathioprine which is a known hepatotoxic agent (15), but conceivably the horse protein could have contributed by the synergistic mechanism described by Paronetto and Popper.

Clinical studies. New patients. -All 8 recipients are alive with excellent renal function from $91 / 2$ to 14 weeks after transplantation. Their courses during the first 63 days of convalescence are compared to those of the 3 earlier groups in Figures 5 and 8.

From 1962 to 1966 a trend to conservatism with the use of azathioprine was noted. The mean daily dose of 1.92 milligrams per kilogram in the fourth series differed significantly from the means of the first and second $(p<0.01)$ but not of the third group. A significant linear downward trend $(p<0.01)$ was present in all.

In contrast, the average daily dose of steroids in the 3 control groups remained quite stable despite the effort to change the therapeutic protocol. However, the patients 


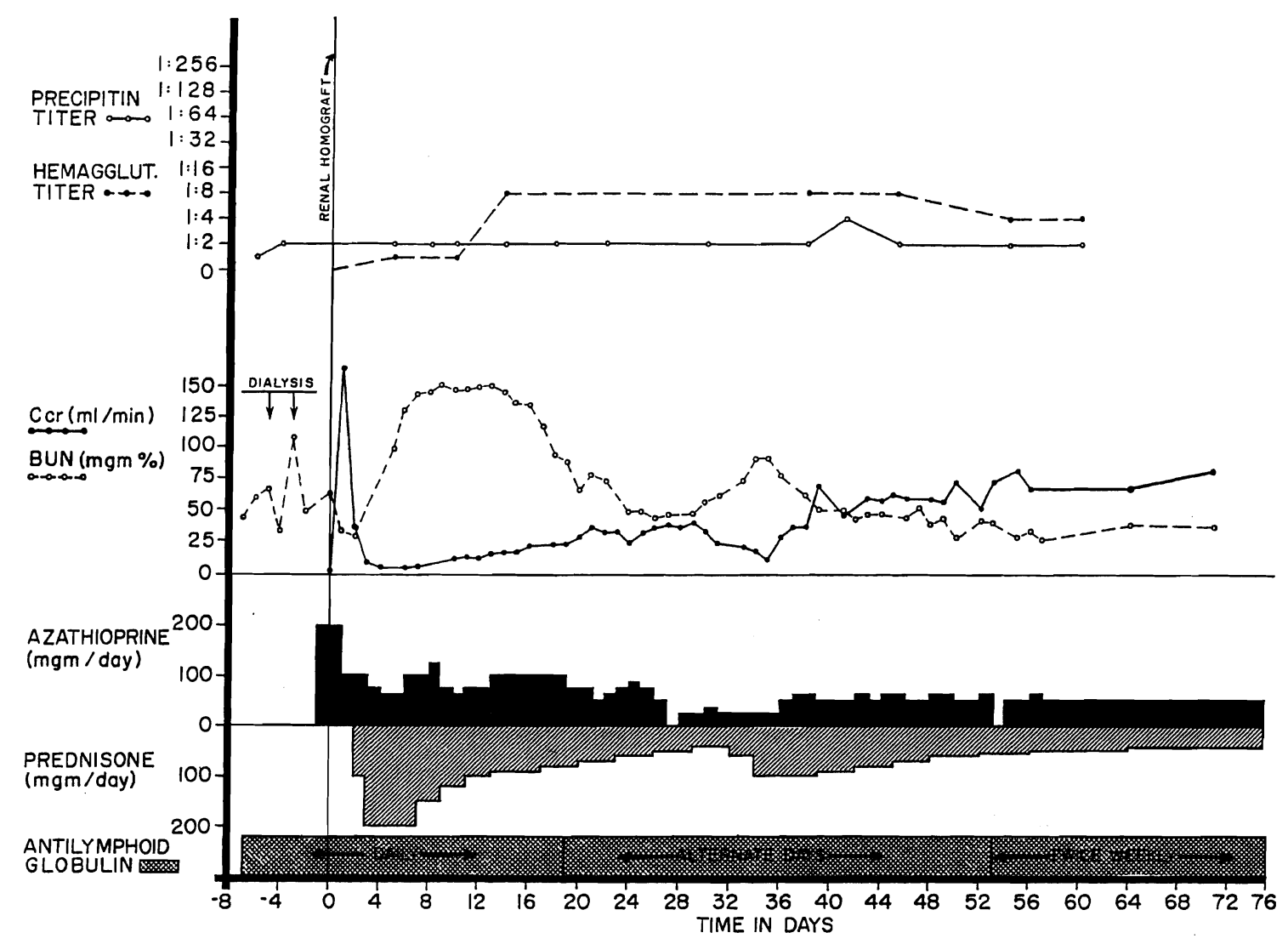

Fic. 7. The course of the only patient who had a moderately severe rejection among the 8 treated from the beginning with antilymphoid globulin $(A L G)$. The young woman, who had a very poor Terasaki antigen match with her fraternal donor, required large doses of steroids which may have contributed to the relative lack of response in the precipitin and leukoagglutinin titers.

in the antilymphoid globulin series received greatly reduced quantities of prednisone. The average daily dose was 0.72 milligram per kilogram, a statistically significant reduction $(\mathrm{p}<0.05)$ compared to 1.58 milligrams per kilogram in the pooled control series.

Renal function in all 4 groups was analyzed to determine if the drug reduction described had been at the expense of an increased homograft injury. When the pooled values of blood urea nitrogen, plasma creatinine, and creatinine clearance from the 3 control series were compared with those of the fourth, there was no significant difference in any of the 3 tested variables.

As would be suspected from these data, difficulties of the antilymphoid series with rejection were not serious. This diagnosis was not made during the first 63 days (Fig. 6) in 4 of the 8 patients. In 3 others, a mild rejection crisis was easily reversed. Only 1 recipient had a secondary rise in blood urea nitrogen to over 100 milligrams per cent, requiring protracted high dose steroid therapy (Fig. 7). These results are compared in Table I with those obtained in the 3 retrospective control series. Only in group 2, in which massive steroid therapy was used from the beginning, was there a similar number of patients who escaped early postoperative rejection. The comparison with group 3 is particularly important. The latter patients all had prospective antigen-typing studies and were treated from the beginning with 30 to 60 milligrams per kilogram per day prednisone, despite which early rejection oc- 


\section{Surgery, Gynecology \& Obstetrics - February 1967}

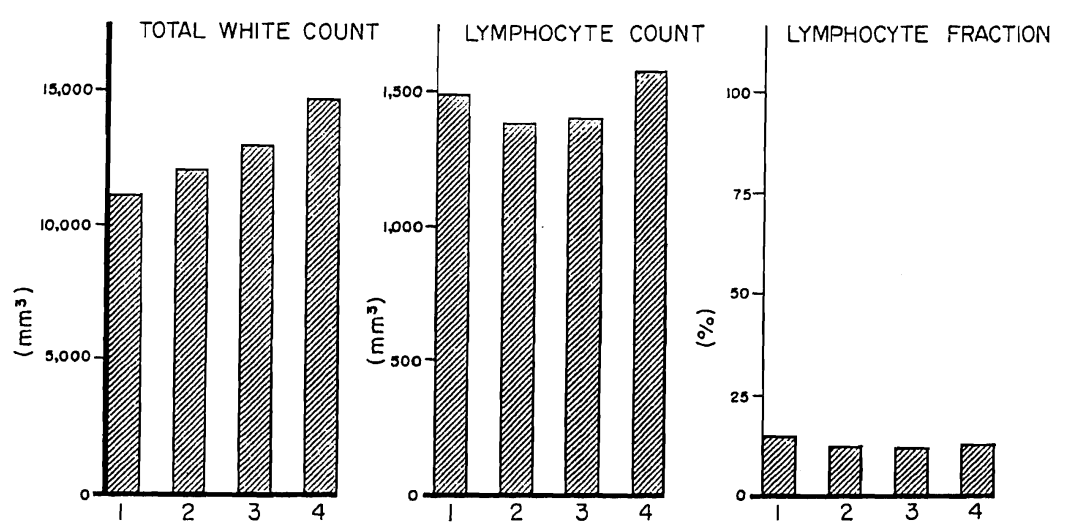

Fig. 8. Effect of different immunosuppressive regimens upon peripheral white cells. The 4 series are in the same order as in Figure 5. Group 4 received adjuvant therapy with antilymphoid globulin. The higher average total white cell count in the later series was apparently due to greater conservatism with the use of azathioprine. The differences in lymphocyte per cents and total counts were not significant.

curred in 82 per cent, necessitating even higher steroid doses.

Blood changes. - These are shown in Figure 8. With succeeding series there was a distinct linear rise in the average daily total white cell count, probably as the result of the increasingly cautious use of azathioprine described earlier. Due to the variability of the white cell counts, the differences between various pairs of groups were statistically nonsignificant. The per cent of lymphocytes in the peripheral blood was approximately the same in all 4 groups. The average total lymphocyte counts ranged from 1,380 to 1,606 per cubic millimeter, the highest values being from the patients receiving antilymphoid globulin (Fig. 8), but the differences were not significant.

Late homograft rejection.-The clinical courses in these 3 patients during the first 10 to 13 weeks of antilymphoid globulin

TABLE I.-INCIDENCE OF REJECTION DURING THE FIRST 63 DAYS IN PATIENTS TREATED WITH ANTILYMPHOID GLOBULIN AND IN THE 3 RET. ROSPECTIVE CONTROL GROUPS

$\begin{array}{llccc} & & \text { No. } & \text { Rejection } & \begin{array}{c}\text { Per cent } \\ \text { rejection }\end{array} \\ \text { Group } 1 \ldots \ldots \ldots \ldots \ldots \ldots \ldots \ldots \ldots & 25 & 23 & 92 \\ \text { Group } 2 \ldots \ldots \ldots \ldots \ldots \ldots \ldots \ldots \ldots & 13 & 7 & 54 \\ \text { Group } 3 \ldots \ldots \ldots \ldots \ldots \ldots \ldots \ldots & 22 & 18 & 82 \\ \text { Group } 4 \text { (antilymphoid) } \ldots \ldots \ldots & 8 & 4 & 50 \\ \text { The reduced number of rejections in group } 2 \text { was evidently due to high } \\ \text { dose prophylactic therapy with prednisone. }\end{array}$

therapy are summarized in Table II. The 2 patients who had received kidneys from related donors had stabilization of renal function despite very drastic decreases in steroid therapy. In both instances, previous dose adjustments of considerably lesser magnitude had resulted in deterioration of function (Fig. 9). The plasma creatinine and the creatinine clearance were not significantly altered in one patient, and in the other there was slight improvement. There were striking falls in the blood urea nitrogen, which were probably at least partially due to decreased protein catabolism secondary to the steroid withdrawal.

The third patient, who had a failing cadaveric homograft, also had a decline in blood urea nitrogen. Nevertheless, there was a measurable further loss of creatinine clearance with a rise in plasma creatinine during the 13 weeks of steroid withdrawal (Table II).

In all 3 patients, the institution of horse globulin reduced the lymphocyte fraction of the differential count but, because the white count was allowed to increase with adjustments of azathioprine dose, the absolute lymphocyte counts were relatively unchanged.

Evidence of serum toxicity. - Fever as well as tenderness at the injection site was seen 


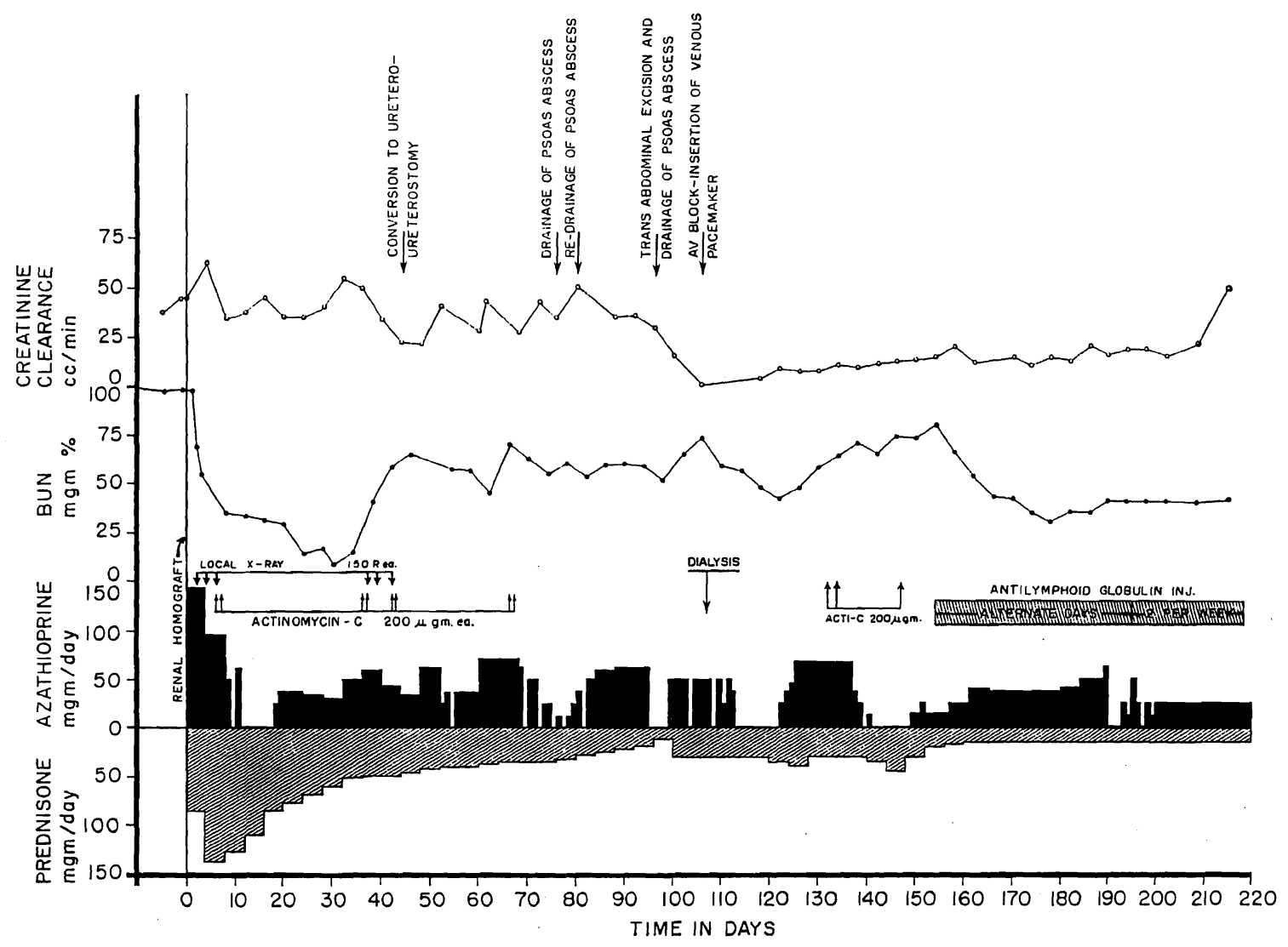

Fig. 9. Course of patient with a failing renal homograft who was treated late with antilymphoid globulin. During the first 4 postoperative months, there were several potentially lethal postoperative complications which were made more grave by the need for heavy steroid therapy. Efforts to reduce the prednisone dosage resulted in deterioration of renal function. After institution of antilymphoid globulin therapy, renal function slightly improved despite a rapid decrease of the steroid doses.

in all patients, most severely after the first few injections.

The antihorse-protein precipitin titers rose slightly in all 11 patients. In 10 the maximum titer did not exceed 1:16 and in the other it reached 1:32. Five patients had a precipitin titer which at first rose and then later declined despite continuation of injections (Fig. 7).

Hemagglutinin titers against sheep red blood cells rose in 8 of 10 patients tested, in 6 instances to $1: 32$ or less, and in the other 2 to $1: 256$ and 1:512. Secondary declines were documented in 4 patients.

Skin tests, which were obtained in 10 patients, showed an increased reaction in 7 . This was usually evident in both the $30 \mathrm{~min}$ - ute and the 24 hour reading and consisted not only of erythema but also of induration.

Four of the 11 patients had a systemic reaction other than fever. In 1 , hives developed. Another became hypertensive for almost an hour. Immediately after injection, in the other 2 patients hypotension developed which lasted for 5 and 30 minutes and which was accompanied by air hunger. All 4 patients recovered without specific therapy and each has subsequently received many more injections without incident.

All-patients had- quantitative studies of urine protein excretion because of recent demonstration in dogs that antilymphoid globulin can cause renal lesions (4). The 3 patients treated late already had proteinuria 


\section{Surgery, Gynecology \& Obstetrics · February 1967}

before instituting globulin therapy and could not therefore be accurately evaluated. None of the 8 patients treated with globulin from the outset has proteinuria judged by standard laboratory urinalysis. Quantitative analysis of 24 hour specimens revealed protein excretion of less than 400 milligrams per day in each after a follow-up of 2 to 3 months.

\section{DISGUSSION}

Two general conclusions with immediate clinical implications seem justified from the information now available concerning antidog-lymphoid products. First, there is an important difference in the response to injections of globulin prepared from the serum of immunized as compared to nonimmunized horses. In the former, development of antibodies to the foreign protein is markedly retarded as measured by the simple and clinically practical precipitin test (4), presumably because of the immunosuppressive qualities of the injectate. The risk of serum sickness is undoubtedly thereby reduced, as the earlier rodent studies of Gray and associates (3) and Levey and Medawar predicted. It is not eliminated, however, since low increases in precipitin titer were often seen in dogs, an observation which may explain the appearance of glomerular lesions after chronic therapy in a significant number of nontransplanted animals as discussed elsewhere (4).

Secondly, although the capacity of the presently available heterologous antilymphoid agents to mitigage rejection of the canine kidney or liver was easily and unequivocally demonstrable in the present series of transplantations, its effect in an individual experiment was unpredictable and weaker than that known to be obtainable with optimal doses of azathioprine. Not only was there a higher incidence of uncontrolled early rejection but also, even after weeks or months, the renal homografts all had evidence of active rejection. It is of interest that more consistent early protection of hepatic homografts was observed, and that in some examined after several months there was little histologic evidence of continuing injury.

The incompleteness of immunosuppression with antilymphoid serum was also evident in the studies of Abaza and his associates with canine renal homotransplantation. Using daily intravenous therapy, they were able to prolong homograft function in 6 of 8 dogs, in 4 of which uremia eventually developed. Maximum survival was 79 days after transplantation and 58 days after removal of the recipient's own kidneys at a second stage operation. Using sheep antidoglymphoid serum intravenously, Mitchell and his colleagues reported only 1 of 8 dogs with survival of more than 22 days, but that animal lived for 10 months after simultaneous homotransplantation and bilateral nephrectomy. The consistency of success has thus been less than that reported in inbred rodent experiments by several previous investigators who employed a skin graft test system.

The foregoing information imposed certain restrictions upon the way in which horse antihuman-lymphoid globulin could be clinically evaluated. It was clear that it had to be used as an adjuvant rather than as the primary immunosuppressive drug. For this reason, it became important to know of the physiologic interaction between the antilymphoid substances and the more conventional immunosuppressive agents. This was a particularly important question since an earlier hypothesis of Levey and Medawar (6), subsequently disproved by the same investigators (7), held that the immunosuppressive properties of antilymphoid serum were due to binding of the heterologous antibodies to recipient lymphocytes thereby "blindfolding" and functionally emasculating them. Such a possibility implied that the action of antilymphoid substances was dependent upon a stabile lymphoid population, and that the superimposition of agents which caused a rapid turnover of stem cells might cancel the effect.

The canine experiments with antilymphoid globulin plus azathioprine were therefore critical. It was found that the limited but definite prolongation of survival, which 
TABLE II.-PATIENTS WITH FAILING RENAL HOMOGRAFTS TREATED WITH ANTILYMPHOID GLOBULIN DURING STEROID WITHDRAWAL

\begin{tabular}{ll} 
Patient & \\
No. & Donor \\
LD 93 & Mother \\
LD 102 & Mother \\
CD 4 & Cadaver \\
\hline
\end{tabular}

$\begin{array}{ccc}\text { Months postop. } & \begin{array}{c}\text { B.U.N., } \\ \text { mgm. per cent }\end{array} & \begin{array}{c}\text { Creatinine, } \\ \text { mgm. per cent }\end{array} \\ \text { ALG started } & 80 \longrightarrow 45 & 2.4 \longrightarrow 2.4 \\ 11 & 80 \longrightarrow 40 & 2.3 \longrightarrow 1.8 \\ 5 & 115 \longrightarrow 75 & 3.2 \longrightarrow 7.0\end{array}$

The changes depicted were over a period of 10 to 13 weeks.

ALG, Antilymphoid globulin.

could be achieved with suboptimal therapy using either agent, was slightly increased when the 2 were employed together. Although this improvement was not statistically significant, the really important observation was that survival was not made worse. Moreover, the extent of histologic damage was less in those homografts from dogs provided with combination therapy.

There is evidence that antilymphoid substances can be used to complement the efficacy of other immunosuppressive measures. The studies which are most relevant to the clinical protocol finally decided upon were made by Levey and Medawar (7). They showed a profoundly synergistic effect of adrenal corticosteroids with antilymphoid serum, an observation not dissimilar to that of Woodruff and Anderson (20) who combined antilymphoid serum with thoracicduct drainage in mice. Levey and Medawar (7) have also demonstrated that total body irradiation and immune serum treatment could be used together with benefit, providing the roentgenotherapy was given before exposure to the antigen. When irradiation was used late, well established skin homografts in serum-treated mice were rejected within a few days, long before the expected time.

The influence of thymectomy upon the effectiveness of antilymphoid serum or globulin therapy is more controversial. Monaco and his associates (10) and Jeejeebhoy, using rabbit antiserums in mice and rats respectively, reported far better results when the thymus had been excised. This could not be confirmed in our canine studies, nor in those of Levey and Medawar (7) whose mouse experiments closely resembled those of Monaco and his associates and of Jeejeebhoy. The performance of thymectomy in some of the patients herein reported may not, therefore, have been justified.

Another detail worth additional comment concerns the proper timing for administration of the antilymphoid substances. Careful studies by Levey and Medawar (6) indicated that 1 or 2 intraperitoneal injections were most effective if given 2 days or more after skin grafting and that pretreatment was of considerably less value. Working with a similar mouse system but with therapy for longer periods, Monaco and his colleagues (9) found pretreatment to be distinctively advantageous, a conclusion supported by our canine studies. There is probably no real inconsistency in these results. Monaco's experiments and those herein reported were not designed to determine the most important time to give a single or a limited number of injections, but instead to determine if treatment for a number of days before as well as after transplantation was superior to that started just before or at the time of operation. It is important to make this distinction, since pretreatment did appear to be of value in the canine experiments and was accordingly made part of the protocol used for patients.

There is evidence that the clinical regimen developed as a result of these various considerations has been at least of short term value. The 8 recipients in the test series did nothave unusually complete antigen matches with their donors and did not appear to enjoy other advantages over previously treated patients. The 8 patients are well from $91 / 2$ to 14 weeks postoperatively. In each of the preceding control series there was a 15 to 28 per cent mortality rate during the same period. The quantities of azathioprine and 


\section{Surgery, Gynecology \& Obstetrics · February 1967}

prednisone were appreciably reduced in the globulin-treated patients during the critical first 2 months without the sacrifice of renal function. This was particularly clear, with calculation of the steroid doses, since the total amounts required in each preceding series had been fixed at almost the same level. The significance of these observations is increased by the fact that data from those patients in the earlier series who died before 9 weeks were excluded from the comparative calculations, thereby introducing a bias against the antilymphoid group.

The influence of the globulin therapy upon the courses of the 3 patients with late-failing homografts is less clear. In 2 of these 3 patients, the doses of prednisone were materially reduced without further evidence of kidney injury. It is probable that renal function in such patients can be improved little, if at all, by institution of antilymphoid globulin injections, but it is possible that residual function can be sustained with considerably less prednisone. Benefit of this sort would not be unexpected even in these patients with late-failing grafts since Monaco and associates (9) and Levey and Medawar (7) showed that antilymphoid serum can erase the immunologic memory of presensitized animals.

The means by which heterologous antilymphoid substances mitigate homograft rejection has been discussed elsewhere from the viewpoint of the pathologic changes they cause in the lymphoid tissues of dogs (4) and in light of the information recently provided by Levey and Medawar (7). Although the immunosuppressive effect of these agents has not been fully explained, the evidence is growing that it is contingent upon neither lymphopenia nor lymphoid depletion. The horse serum or globulin used for dogs and patients of the present study caused lymphopenia under appropriate conditions of in vivo testing (4), a fact which is probably of practical importance in deciding whether or not a potent product is being used. Nevertheless, this was often not observed in animals which survived for long periods after either kidney or liver homotransplantation.
In the patients who were treated with 2 or more drugs in addition to globulin, the significance of changes in lymphocyte counts was even more difficult to interpret, particularly when comparing results in the test group with those obtained from earlier series. In a past clinical study which included many patients from the retrospective control groups (16), it was shown that lymphocytosis in the post-transplant period could not be correlated with the presence or absence of rejection. There was, however, a direct correlation between the quantity of steroids employed and the degree of lymphopenia. This important pharmacologic variable was present in both our control and test series. Consequently, more cannot be said than that the patients treated with antilymphoid globulin had approximately the same number of peripheral lymphocytes as previously treated patients who had received more than twice as much prednisone.

Thus far, discussion has been largely confined to the considerable body of evidence supporting the concept that heterologous antilympoid globulins may be useful agents in clinical homotransplantation. It is important to emphasize that the extent to which this may be true will be strongly influenced by further studies of clinical toxicity. It was mentioned earlier that glomerular lesions can be produced in normal dogs by chronic administration of these substances. Biopsy of the human homografts will be necessary at a relatively early time to determine if similar lesions are present, even though none of the patients now has proteinuria. Such histologic information will guide a decision for or against further therapy in these or future patients.

Furthermore, other features of the patients' responses to horse protein are not yet clear. The modest and often reversible increases in antihorse-precipitin titers have been cited. Moreover, the significance of rises in antisheep red cell agglutinins, which are apparently in response to a Forssman type antigen in the horse protein, is not yet known. Although life-threatening toxicity has not been 
observed, this possibility has not yet been excluded. It was principally because of this consideration that 4 of the 8 patients in the series of new patients were eventually treated with low dose steroid therapy even though the clinical diagnosis of homograft rejection had not been made.

\section{SUMMARY}

Plasma, serum, or globulin were prepared from the blood of horses which had been immunized with canine lymphoid tissues. The respective agents which were rendered progressively less toxic by appropriate absorption and purification procedures were administered by the intraperitoneal or subcutaneous route to dogs which received either renal or orthotopic liver homotransplantation. The survival of animals after either kind of procedure was significantly prolonged compared to that of controls, although in virtually all clinical and pathologic evidence of rejection eventually developed. The best results were obtained if treatment was started several days before operation and continued thereafter. The degree of protection seemed to be greater for the liver homografts than for the kidneys. Combination therapy of antilymphoid globulin and small doses of azathioprine resulted in slight and statistically nonsignificant increases in survival, but the degree of histologic injury seemed to be reduced.

Although easily demonstrated, the immunosuppressive effect of the antilymphoid products was not strong enough to warrant clinical use as other than an adjuvant agent. Horse antihuman-lymphoid globulin has been used for this purpose in 11 patients in combination with azathioprine and prednisone. Eight of these patients received intramuscular globulin starting before operation and continuing until the present time. Their need for therapy with the standard immunosuppressive drugs has seemed to be reduced without a consequent loss of homograft function. All are well after $91 / 2$ to 14 weeks. The other 3 patients had failing renal homografts which had been placed from 5 to 11 months previously. After institution of globulin therapy in the latter patients, steroid doses were reduced. In 2 of the 3 patients, renal function stabilized or improved in the ensuing 10 to 13 weeks, but in the third there has been further deterioration.

The experimental nature of the clinical trial has been stressed in light of the unknown risk of serious toxicity from the injections of horse protein. This morbidity can be completely assessed only with further observation and by study of homograft biopsies in those patients now under treatment. Wider clinical trial is not recommended until this information has been obtained.

\section{REFERENCES}

1. Abaza, H., Nolan, B., Watt, J., and Woodruff, M. F. A. The effect of antilymphocytic serum on the survival of renal homotransplants in dogs. Transplantation, 1966, 4: 618.

2. Gray, J. G., Monaco, A. P., and Russell, P. S. Heterologous mouse antilymphocyte serum to prolong skin homografts. Surgical Forum, Clinical Congress 1964. Vol. XV, p. 142. Chicago: American College of Surgeons, 1964.

3. Gray, J. G., Monaco, A. P., Wood, M. L., and RUsseLI, R. S. Studies on heterologous antilymphocyte serum in mice- $I$, in vitro and in vivo properties. J. Immun., Balt., 1966, 96: 217.

4. IWASAkI, Y., PORTER, K. A., AMEND, J., MARCHIORO, T. L., ZüHLRE, V., and STARZL, T. E. The preparation and testing of horse antidog and antihuman antilymphoid plasma or serum and its protein fractions. Surg. Gyn. Obst., 1967, 124: 1.

5. JEEJEEBHOY, H. F. Immunological studies on the rat thymectomized in adult life. Immunology, 1965, 9: 417 .

6. Levey, R. H., and Medawar, P. B. Some experiments on the action of antilymphoid antisera. Ann. N. York Acad. Sc., in press.

7. Idem. Nature and mode of action of antilymphocyte antiserum. Proc. Nat. Acad. Sc. U. S., in press.

8. Mrtchell, R. M., SheIr, A. G. R., SlafsKy, S. F., and Murray, J. E. The effect of heterologous immune serum on canine renal homografts. Transplantation, 1966, 4: 323 .

9. Monaco, A. P., WOOD, M. L., Gray, J. G., and RUSSELI, P. S. Studies on heterologous antilymphocyte serum in mice-II, effect on the immune response. J. Immun., Balt., 1966, 96: 229.

10. Monaco, A. P., Wood, M. L., and Russell, P. S. Effect of adult thymectomy on the recovery from immunologic depression induced by heterologous antilymphocyte serum. Science, 1965, 149: 423.

11. NAGAYA, H., and SIEKER, H. O. Allograft survival; effect of antiserum to thymus glands and lymphocytes. Science; 1965, 150:-1181.

12. Paronetro, F., and POPPER, H. Aggravation of hepatic lesions in mice by in vivo localization of immune complexes. Am. J. Path., 1965, 47: 549.

13. Porter, K. A., CalNe, R. Y., and Zukoskr, C. F. Vascular and other changes in 200 canine renal homotransplants treated with immunosuppressive drugs. Laborat. Invest., 1964, 13: 809. 
18 Surgery, Gynecology \& Obstetrics - February 1967

14. Starzl, T. E., Marchioro, T. L., Faris, T. D., MCCARDIE, R. J., and IWASAKI, Y. Avenues of future research in homotransplantation of the liver; with particular reference to hepatic supportive procedures, antilymphocyte serum and tissue typing. Am. J. Surg., 1966, 112: 391.

15. Starzi, T. E., Marchioro, T. L., Porter, K. A., TAYLOR, P. D., Faris, T. D., HerrmanN, T. J., HLAD, C. J., and WADDELI, W. R. Factors determining short- and long-term survival after orthotopic liver homotransplantation in the dog. Surgery, 1965, 58: 131 .

16. Starzl, T. E., Marchioro, T. L., Terasaki, P. I., Porter, K. A., Faris, T. D., HerrmanN, T. J., VRedevoe, D. L., HutT, M. P., Ogden, D. A., and WADDELL, W. R. Chronic survival after human renal homotransplantation. Ann. Surg., 1965, 162: 749.

17. Terasaki, P. I., Porter, K. A., Marchoro, T. L., Mrckey, M. R., VRedevoe, D. L., Faris, T.

D., and StarzL, T. E. Serotyping for homotransplantation-VII, selection of kidney donors for 32 recipients. Ann. N. York Acad. Sc., in press. 18. Waksman, B. H., Arbouys, S., and Arnason, B. G. The use of specific "lymphocyte antisera" to inhibit hypersensitive reactions of the delayed type. $J$. Exp. M., 1961, 114: 997.

19. WoOdRUFF, M. F. A. The Transplantation of Tissues and Organs. Pp. 100, 150. Springfield, Ill.: Charles C Thomas, 1960.

20. Woodrufr, M. F. A., and Anderson, N. F. Effect of lymphocyte depletion by thoracic duct fistula and administration of antilymphocyte serum on the survival of skin homografts in rats. Nature, Lond., 1963, 200: 702.

21. Idem. The effect of lymphocyte depletion by thoracic duct fistula and by the administration of antilymphocyte serum on the survival of skin homografts in rats. Ann. N. York Acad. Sc., 1964, 120: 119. 